\title{
When is a Lattice Not a Lattice? The changing meaning of the term lattice in crystallography and physics
}

\author{
Robert G. Palgrave ${ }^{\mathrm{a}}$ and Emma Tobin ${ }^{\mathrm{b}}$
}

\begin{abstract}
a) Department of Chemistry, University College London, 20 Gordon Street, London WC1H $0 \mathrm{AJ}$

b) Department of Science and Technology Studies, University College London, 22 Gordon Square, London WC1H 0AW
\end{abstract}

email: r.palgrave@ucl.ac.uk,e.tobin@ucl.ac.uk

\begin{abstract}
The concept of the lattice is central to the understanding of crystalline solids. However, usage of this word can very often differ between crystallographers, for whom a lattice is a mathematical object that describes the symmetry of a crystal, and physicists and chemists, for whom lattice is commonly used as a word for a regular array of particles. The similarity between the two definitions means they can easily be confused by students or inexperienced practitioners, but the fundamental differences can make the consequences of such confusion significant, an issue rarely tackled directly in popular textbooks. Here we examine the historical roots of this problem of context, and the changing understanding of the word 'lattice' over time. While the origins of the term lattice lie with the $19^{\text {th }}$ century mathematical crystallographers, their usage, both in terms of the words used and their meanings, was fluid, and no strong distinction between the lattice and the physical components of a crystal was made. Leading crystallographers in the early $20^{\text {th }}$ century regularly used the word lattice in a way that is unacceptable to some of their modern counterparts. We identify the decade after 1910 as the start of divergence between the physical and crystallographic meaning, catalysed by the discovery of X-ray diffraction and the development of lattice dynamics, although the current definitions did not become entrenched until the 1940s. While history has shown us that this classificatory issue is not just a matter of scientific disagreement and perhaps at its root is a disagreement in our understanding of classification itself. Lastly, we discuss possible resolutions to the matter.
\end{abstract}




\section{Introduction}

Classification is important in the physical and mathematical sciences. It allows scientists to group the objects of inquiry into classes. The aim of division into these classes is to impose an order and categorisation, which makes our knowledge more parsimonious, facilitates generalisation and prediction, and at a basic level makes communication possible between scientists. A simple example of where classification is effective is Mendeleev's periodic table. There is a unitary principle for classification, namely the atomic number.

The term lattice is used across a wide variety of scientific fields, often in the context of the study of crystals. Crystals consist of an ordered arrangement of particles (atoms, ions or molecules) which usually possesses translational symmetry. While crystals have been of interest to humankind since prehistory, in the past two centuries their study has transformed our conceptions of matter and driven many technological advances without which the modern world would be unrecognisable. The term lattice is central to the study of crystals, but there are two distinct and well established meanings of lattice in use in current scientific practice. One definition of a lattice is understood in abstract terms, defined mathematically as a set of points, and operating as a descriptor of symmetry in a crystal structure. Conversely, in solid state physics and chemistry, a lattice is often defined as a periodic arrangement of particles as is found in a crystal. For many physicists and chemists, it is this physical arrangement of the particles that is the lattice. Wong et al. describe situations such as this as 'problems of context', where different technical fields use the same word in different ways. ${ }^{1}$ Many other examples of such problems exist, including such seemingly fundamental terms as heat, magnetic field, or molecule, which are each defined differently in different technical contexts. ${ }^{1,2}$

In this article we first set out the differences between the definitions of lattice, and the implications of the current dual usage. We then describe both the historical development of the word lattice, and that of the underlying concepts behind the two definitions. We attempt to identify the historical point of divergence in meaning, and in the final section, we point to some of the possible resolutions of the current situation. 


\section{The Word 'Lattice' and its Meanings: Crystallographic Lattice and Physical Lattice}

The word lattice has two well established meanings within the community of scientists working on crystalline materials. The first, which was provided recently by Nespolo ${ }^{3}$ and can be found in every contemporary crystallographic textbook, we will refer to as the crystallographic lattice. In short, the crystallographic lattice is a set of points, called lattice nodes (or sometimes lattice points), defined by integer sums of a set of linearly independent vectors. There are exactly 14 types of lattice in three dimensional space. A notable feature of this definition is that it is an entirely abstract concept; the crystallographic lattice is not a physical object, but an infinite set of points in space. The physical components of a crystalline object can be thought of as arranged in space by some relationship to the lattice. To take the simplest example, the element gold $(\mathrm{Au})$ adopts a structure that can be thought of as follows: imagine a face centred cubic lattice (which is one of the 14 previously mentioned types) and then place one atom of $\mathrm{Au}$ on each lattice node. What you have now is the ideal crystal structure (or crystal pattern) of $\mathrm{Au}$, which is an infinite array of Au atoms arranged in a one-to-one correspondence with the lattice nodes. This one-to-one correspondence of lattice point and atom is certainly not a requirement, as can be seen in the crystal structure of diamond. Here, each lattice node coincides with a carbon atom, but that only accounts for half of the atoms in the structure: there is also a second set of carbon atoms at the centre of tetrahedra formed by the atoms on the lattice nodes: half of the carbon atoms do not coincide with lattice nodes at all. In fact, all the carbon atoms in diamond have exactly equivalent surroundings, and we could just as well have chosen to move the lattice so that the nodes coincide with the second set of carbon atoms. It might be tempting to think that diamond should therefore be described by two lattices, but that is not correct. Diamond, like every crystal, has only one crystallographic lattice, which describes the overall symmetry, but in diamond there are two atoms associated with each lattice node. 

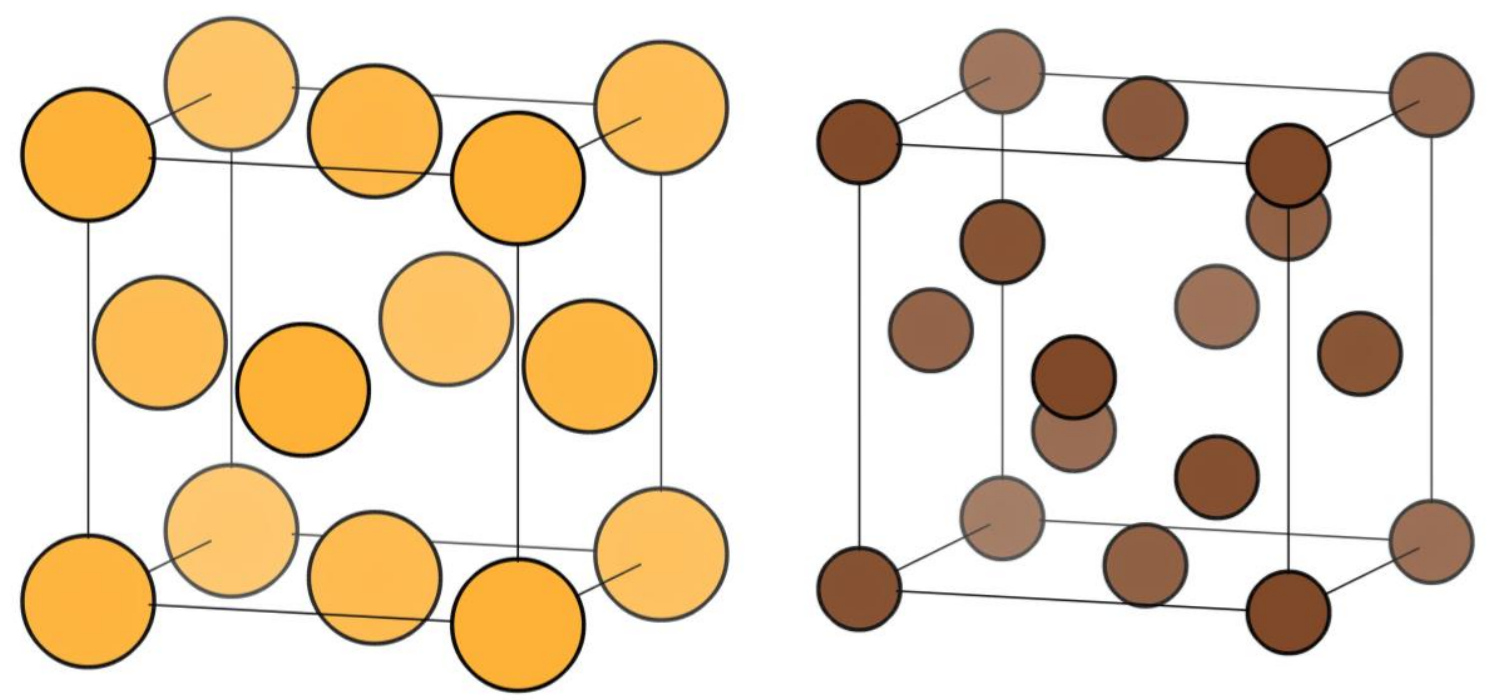

Figure 1. The crystal structure of gold, left, and diamond, right. Both have a face centred cubic lattice. There is a one-to-one correspondence between gold atoms and lattice nodes. In diamond only half of the carbon atoms are on lattice nodes. On solving the diamond structure in 1913, Lawrence Bragg described diamond as having "two interpenetrating face-centred space lattices", ${ }_{4}$ a description at odds with current crystallographic usage.

Of course, a real crystal of gold or diamond will differ from the ideal crystal structures just described in many important ways, not least of which is that any real crystal is finite in size. It will also have surfaces and will in almost all cases include static defects (e.g. point vacancies, dislocations etc) and in all cases dynamic defects (phonons): none of these are described by the ideal crystal structure. Despite this, some important properties of a solid can be easily deduced, at least approximately, from the ideal crystal structure, for example density, bond lengths, and coordination number of the constituent atoms. Many other properties can be calculated using quantum mechanical approaches using the ideal crystal structure as a starting point, such as the formation energy of the crystal, electrical and thermal conductivity, the energies of the bound electrons, the phonon energies etc.

The key point, however, is that the crystallographic lattice is not the crystal; it is not a physical object, but rather an abstract descriptor of the crystal symmetry, and so it has none of the physical properties listed above. Thus, terms such as lattice defect are not meaningful, since an abstract mathematical object cannot have defects. The terms lattice energy, lattice vibration, lattice dynamics and lattice strain are also contradictions in terms. ${ }^{5}$ 
The second definition of lattice can be summed up by Max Born and Kun Huang, in their 1954 book, Dynamical Theory of Crystal Lattices, which states that

"Crystalline solids are distinguished from other forms of matter by a periodic arrangement of atoms; such a structure is called a crystal lattice" 6

In this meaning, lattice is the word to describe the regular arrangement of atoms, molecules or ions that might be found in a crystal. For the purposes of this article, we will refer to the kind of lattice described in the preceding quotation as a physical lattice, to emphasise the main conceptual difference between this definition and the crystallographic lattice; since it is a periodic arrangement of atoms, ions or molecules as found in a crystal, a physical lattice is a physical object, it has properties such as density, conductivity, internal energy, etc, and can vibrate, expand, contract, deform etc. Many other scientific phrases in very common use in chemistry and physics include the word lattice and can only make sense if the lattice in question is a physical lattice. For example, lattice solvent is defined as "uncoordinated solvent molecules occupying well defined positions in [a] crystal". ${ }^{7}$ Lattice thermal conductivity is one part of the thermal conductivity in a crystal, the other being the electronic thermal conductivity. ${ }^{8}$ The lattice energy is a central concept in the thermodynamics of solids, and in this phrase the lattice must be physical (or it could not have an energy). ${ }^{6}$

In modern chemistry and physics textbooks, often the distinction between physical and crystallographic lattice is unspoken, but implied. A. West in Basic Solid State Chemistry (1984 and subsequent editions) carefully defines a lattice point in agreement with the crystallographic definition, but then also uses the word lattice clearly to mean a physical thing, e.g. in the discussion of lattice imaging (by electron microscopy) and the lattice energy. The International Union of Pure and Applied Chemistry (IUPAC) does not define lattice specifically, but uses the word lattice to mean physical lattice in a number of other definitions, for example the definition for superlattice, phonon ("vibrations in a crystal lattice") and lattice distortion. Despite the widespread use of the dual meanings of lattice, it seems rare for authors of modern textbooks to confront the matter directly. An exception is S. H. Simon who expressed the existence explicitly of dual meanings of the word lattice: 
"Warning: Some books... refer to this [crystallographic lattice] as a Bravais lattice. This enables them to use the term lattice to describe other things",

the 'other things' being physical objects.

In this paper we seek to explore the historical origins of the dual meaning of lattice, and then to suggest possible resolutions to the issue. A detailed history of crystallography is well beyond the scope of this article, but a short introduction to the main themes is important for the discussion that is to follow. For more comprehensive accounts, the reader is directed to several books $^{10-12}$ and journal articles ${ }^{13-17}$ on the topic.

The concept that a crystal has a regular internal arrangement that bears some relationship to its macroscopic form can be tentatively traced as far back as ideas of ancient Greek atomic theory. ${ }^{18}$ Certainly Newton, Kepler and Huygens sought to explain macroscopic crystal forms by reference to an internal microscopic arrangement. The mathematical science of crystallography, the effort to understand the possible arrangement of three dimensional patterns with translational symmetry, might be said to begin in earnest at the start of the $19^{\text {th }}$ century with the work of René Just Haüy. After a century of progress, Hilton was able to state in 1903 that "The geometrical theory of crystal-structure now seems to be fairly complete" ${ }^{19}$ It is easy to understand this confidence; Bravais had derived the 14 possible types of three dimensional lattices in 1848, and later Schoenflies, Fedorov and Barlow had identified the 230 space groups, specifying every way that objects of any symmetry may be arranged in any possible 3D repeating pattern. Yet as the $20^{\text {th }}$ century began, the practical application of these mathematical methods was much more limited. Before the watershed discovery of X-ray diffraction in 1912, it was not possible to assign a space group, nor even in many cases a Bravais lattice, to a particular crystalline material with any reliability. ${ }^{13}$ The lattice theory, the idea that crystals were made up of regularly spaced physical elements, had suffered important practical setbacks, in that Cauchy's predictions of elasticity of crystals, which were based on lattice theory, had been shown to be incorrect in $1848 .{ }^{20}$ Thus while the mathematics of lattices and space groups developed apace, its power to affect the physical sciences lay dormant, until eventually unlocked by two key moments. Firstly, as already mentioned, the discovery of X-ray diffraction by Max von Laue and co-workers in 1912, which would not only prove beyond doubt the lattice theory of crystals, but allow for the first time direct measurements of the arrangement of atoms in a substance. Secondly, around the same time, the development of lattice dynamics by Max 
Born and others, which successfully married the idea of the lattice with the emerging quantum theory to yield what remains the overarching framework of solid state physics. These two developments would cause the study of crystals to move in a relatively short time from a niche topic that bridged applied mathematics and parts of geology and minerology, to a central discipline in modern physical science, underpinning a vast range of work in physics, chemistry and, eventually, biology. We will go on to argue later in this article that the dual use of the term lattice that we find today can be traced back to these two almost simultaneous developments.

Today, proper understanding of the crystallographic lattice is essential for all those who would use the methods of crystallography to whatever degree. The pitfalls of confusing lattice with structure, lattice nodes with atoms, can be severe, from misunderstanding other scientists, to erroneous input into (sometimes very costly) calculations, to misinterpretation of diffraction patterns. The potential for confusion is increased as the issue of the dual meaning of lattice is often not directly addressed in physics and chemistry texts, despite the fact that the existence of dual meanings has been highlighted for many years. For example, in 1942, M. J. Buerger wrote:

"Many who have written about crystal structures, particularly chemists, physicists and metallurgists, unacquainted with pattern theory, have either used the term lattice incorrectly, or thought to extend its use to mean the actual material crystal structure of packed atoms." 21

By pattern theory, Buerger is referring to the mathematical theories of crystallography developed in the $19^{\text {th }}$ century. Nespolo has recently highlighted a similar complaint, with a detailed description of the crystallographic meaning of lattice and how it contrasts with some current usage. ${ }^{3}$ Nespolo describes the use of terms such as lattice energy, lattice dynamics etc, and states:

"What the writer has in mind is obviously the structure, but because of an incorrect shortcut, he calls it lattice." 5

As shown in the quotations from Buerger and Nespolo above, it is sometimes asserted that the crystallographic definition of lattice is the 'correct' one, and that use of lattice in a way as to imply a physical object is erroneous. Nespolo justifies this as follows: 
"terms like lattice and structure have been defined on an international basis by the IUCr [International Union of Crystallography], in much the same way as most other physical terms are. For instance, we would not be allowed to use, say, $g$ the acceleration due to gravity, as the same as the force due to gravity."

This places the justification for the primacy of the crystallographic definition in the definition by IUCr, the international body founded in 1946 to promote international cooperation in crystallography. As seen in the quotation above, Buerger, writing just before the foundation of the IUCr, instead blamed ignorance of underlying mathematical theory on the incorrect use of lattice.

We will now critically assess these claims by surveying the historical usage of terms in crystallography, physics and chemistry.

\section{The emergence of the physical lattice}

Firstly, we will deal with the historical use of the word lattice to mean a physical object, a physical lattice. The use of the term lattice by physicists might be conservatively dated to the first two decades of the $20^{\text {th }}$ century. As mentioned, the field of lattice dynamics was founded by Born, Madelung, Karman and others from 1909 onwards, ${ }^{22}$ as an attempt to understand the properties, especially vibrational and thermodynamic, of crystalline solids in terms of their atomic structure. In 1915, Born published his first book, Dynamik der Krystalgitter (Dynamics of Crystal Lattices), and in 1918, with Lande, introduced the idea of Gitterenergie, or lattice energy. ${ }^{23}$

Much later, Born recalled that even though this work on Gitterdynamik, lattice dynamics, was started before Laue's 1912 discovery of X-ray diffraction "we regarded the existence of lattices as evident [because] we knew the group theory of lattices of Schoenfiles and Fedorov"24

The theory that Born refers to here is the work that Schoenfiles and Fedorov had independently (and later collaboratively) carried out to list the 230 space groups - the different ways of arranging an object (such as a molecule) of any symmetry on the nodes of a lattice. Born and the other pioneers of lattice dynamics were not the first to consider the vibrational properties 
of solids. Einstein and Debye, for example, both produced models that matched well with experimental heat capacities in the early $20^{\text {th }}$ century. ${ }^{25,26}$ But neither of these models used a realistic picture of the crystal structure of the solids $;{ }^{27}$ Born and his collaborators were the first to successfully combine the ideas of symmetry from mathematical crystallography with the new ideas of atomic structure and Planck's quantum radiation formula; the result was a theoretical framework that has dominated solid state physics and chemistry ever since. ${ }^{10}$ The ideas introduced as a result: lattice energies, periodic boundary conditions, normal and characteristic modes of vibration, are all of enduring importance in understanding physical properties of crystalline materials today. ${ }^{28}$ For this reason, we take this body of work in the 1910s to be the moment of definition of lattice understood as a physical lattice and set the scene for the usage of lattice by physicists.

We can now pose the question: at the time Born and co-workers were establishing lattice dynamics, what terminology was used by crystallographers concerning the crystallographic lattice? At the time of their work, were Born and his contemporaries incorrect to use terms like Gitterenergie and Gitterdynamik as they did, imparting physical properties to the Gitter, the lattice? To answer this question, we turn to the work of crystallographers of the same period and preceding decades.

Let us first consider the work of the German school of crystallographers in the period around the first decades of the $20^{\text {th }}$ century. Paul von Groth was the founder of the world's principal journal of crystallography of the time, the Zeitschrift für Krystallographie und Mineralogie which he edited from 1877 to 1920. The journal published many important papers in German, such as Fedorov's deviation of the space groups and Barlow's prediction of the crystal structure of $\mathrm{NaCl}^{29}$ Groth also wrote five volumes of Chemische Kristallographie, which listed the crystallographic properties of nearly 10,000 substances. He was described in 1912 as the 'doyen of the crystallographic world' by the English crystallographer Tutton, ${ }^{30}$ and later by P. P. Ewald as "champion and chief authority of crystal structure." 13 Thus at the start of the $20^{\text {th }}$ century, on the eve of the discovery of X-ray diffraction which would radically transform his field, Groth was perhaps the foremost crystallographer in the world. In 1921 Groth published his final textbook, entitled Elemente der physikalischen und chemischen Krystallographie (Elements of Physical and Chemical Crystallography), and there he describes the freezing of a liquid to a solid as follows (page 2): 
“die bis dahin voneinander unabhängig bewegten Moleküle sich zu einem Atomgitter d.h. zu einer regelmäßigen Anordnung von Atomen vereinigen"

"the molecules that had previously moved independently of one another form an atomic lattice i.e. a regular arrangement of atoms"

Here we see that Groth is happy to use Atomgitter, (atomic lattice) and helpfully defines this as a regular arrangement of atoms, in a crystal. He introduces Bravais lattices like so:

"In strengerer Weise bewies Bravais 1848, daß es vierzehn mögliche Arten von Raumgittern gebe"

"In 1848, Bravais more rigorously proved that there were fourteen possible types of space lattices"

Here the term Raumgitter, (space lattice) is used to describe the lattices enumerated by Bravais. The term Raumgitter was first introduced by Sohncke, the classifier of space groups, in 1874, with the same meaning. ${ }^{31}$ It was used by Fedorov when writing in German, ${ }^{32}$ in fact, we find that space lattice was the phrase generally used for a Bravais lattice by the community of German speaking crystallographers working in the first decades of the $20^{\text {th }}$ century. It might be argued that the prefix Raum- in Raumgitter (space in space lattice) was used to denote a crystallographic lattice in three dimensions, as opposed to two or one dimensions. This would make sense if lower dimensional lattices also had a different prefix appended to Gitter. However, that is not the case. A two dimensional lattice in German was called Punktnetz or Netzebenen, and a one dimensional lattice was a Punktereihe. ${ }^{19,33}$

At the same time that Groth was writing his final textbook, P.P. Ewald, was writing his first. In 1923, Ewald was a young scientist who, having played a role in the discovery of X-ray diffraction, would go on to become one of the $20^{\text {th }}$ century's foremost crystallographers, and one of the co-founders and a president of the IUCr. Ewald's textbook in 1923 was entitled Kristalle und Röntgenstrahlen, (Crystals and X-rays). ${ }^{33}$ He uses the phrase Atomgitter over 35 times - for example, describing the X-ray diffraction pattern as (p. 69): “Wie ein Schattenriß des Atomgitters", "like a silhouette of the atomic lattices" 
Elsewhere, discussing zinc blende (p.96), Ewald talks of "atomic lattices made of Zn and S", and when discussing diffraction, Ewald states (p. 40) "Let us now imagine the space lattice of a crystal hit by a wave. Each lattice point, i.e. every atom becomes the starting point of a spherical wave." Not only is the lattice point identified as equivalent to an atom, but it is said to interact directly with an electromagnetic wave. Recall that the phrase Atomgitter, atomic lattice, and the idea that the lattice is a physical thing are anathema to the modern crystallographic definition, which holds a lattice to be an entirely abstract concept.

These examples show that leading crystallographers even as late as the 1920s used Gitter in a way that clearly meant a physical thing. To further examine the usage of the word Gitter, we have reviewed all the papers published in Zeitschrift für Krystallographie und Mineralogie in 1911 and 1914. These dates were chosen to be immediately before and after the discovery of $\mathrm{X}$-ray diffraction, and also contemporary with the important publications in lattice dynamics referenced above. We found no example where the word Gitter, used alone, could conceivably mean crystallographic lattice; all instances were clearly referring to a physical rather than abstract object. We found that every instance of the use of the word Raumgitter could be best understood as meaning Bravais lattice. While it is absolutely clear from the work of these German crystallographers that Gitter can mean a physical object, the relationship between the Raumgitter and the physical components is less clear, but we will defer this question to the next section, as it does not affect our immediate conclusion. From the textbooks written by Groth and Ewald in the 1920s, and from the papers published in the leading crystallography journal of the day, we see that in the German language crystallographic literature, Gitter certainly meant a physical object. Phrases such as Atomgitter (atomic lattice) were acceptable to the leading German speaking crystallographers in the early $20^{\text {th }}$ century. This use of language seems to us entirely in accord with the terminology used by Born and other physicists working on lattice dynamics. If Atomgitter is permitted by German speaking crystallographers in 1920, then Gitterenergie and Gitterdynamik must also be consistent with their definitions. We conclude that no mistake in terminology was made by Born in producing his lattice dynamics. 
Of course, not all crystallography was reported in German, and by the time of Groth and his contemporaries, over a century mathematical crystallography texts had been written in French and Russian, as well as German, with a much more limited amount in English. We have discussed the language of the German speaking crystallographers in the first two decades of the $20^{\text {th }}$ Century. Now we will attempt to understand the usage of lattice and related terms in French and English, in the $19^{\text {th }}$ century.

Table 1. Comparison of terms for crystallographic lattice, as reported by Hilton, 1903, and as currently recommended by the International Union of Crystallography (IUCr).

\begin{tabular}{|c|c|c|}
\hline & Hilton, $\mathbf{1 9 0 3}$ & Modern IUCr term \\
\hline English & lattice & lattice \\
\hline German & Raumgitter & Gitter \\
\hline French & assemblage & réseau \\
\hline
\end{tabular}

A starting point is Hilton, who, in writing the first textbook in English on mathematical crystallography in 1903, provides a list of equivalent terminology in English, French and German. ${ }^{19}$ This is reproduced in Table 1, along with the modern $\mathrm{IUCr}$ approved terms in the three languages. Hilton also gave a short comment on this terminology. He stated:

The words lattice, assemblage, Raumgitter are not satisfactory; 'assemblage' is too vague, while 'lattice'suggests a plane figure. We have, however, employed it [lattice] as it is shorter than 'space lattice', although the latter is in more general use.

Indeed, Hilton is correct in that Bravais himself had used assemblage to mean lattice, and in fact he used réseau (the modern French word for lattice approved by IUCr) to mean a two dimensional net. Other writers in French, however, preferred to use réseau to mean three dimensional lattice, as is the case in modern French. Delafosse, whose work came before Bravais, used réseau, ${ }^{34}$ as did Mallard, who came after. Assemblage did cross over into English however, no doubt due to the influence of Bravais' work. Kelvin uses the word 'assemblage' to describe the arrangement of molecules in a crystal, which he ascribes to Bravais, to whom, according to Kelvin, is owed "the whole doctrine of homogeneous assemblages in its most perfect simplicity and complete generality." ${ }^{35}$ Interestingly, Kelvin, in his lectures, used an actual wooden lattice, which he could distort to demonstrate various planar symmetries, in 
dimensional accord at least with Bravais' terminology. Barlow, who separately to Fedorov enumerated the space groups pioneered the idea of building models to understand crystal structures, and used the word assemblage to describe these models, ${ }^{36}$ again blurring the line between the abstract and real.

Hilton's is the first major text in English on mathematical crystallography. As discussed, he advocated the term lattice over space lattice, due to brevity. As it turned out, it was several decades before this suggestion was adopted by English speaking crystallographers. As we have seen, Bravais' assemblage had found its way into English in the works of Kelvin, Barlow and others. By the first decades of the $20^{\text {th }}$ century, however, virtually all English language crystallographic writing used the term 'space lattice' in exactly the same way that the German school did with the term Raumgitter, and this continued for some time despite Hilton's recommendation. In his later writings, Barlow too used space lattice in this way, ${ }^{37}$ and this passage from W.L. Bragg, in his description of diffraction in 1913, explicitly identifies space lattice with Bravais lattice: ${ }^{38}$

In treating the diffraction of waves by a space point system such as a crystal, that case is the most simple in which the diffraction is caused by a series of points arranged in a space lattice, of one of the 14 Bravais types.

W.L. Bragg repeats this definition, of space lattice being equivalent to three dimensional Bravais lattice, in his final book, The Development of X-ray Analysis, which was completed shortly before his death in 1971, showing he maintained this definition his whole career. ${ }^{39}$

We have attempted to build a picture of the use of language by crystallographers in the early $20^{\text {th }}$ century, at the time that physicists and chemists began to seriously use ideas of the physical lattices in their theories. The German school of crystallographers, exemplified by Groth and Ewald, used Raumgitter with a meaning closest to the modern crystallographic lattice, and Gitter to mean a physical object. Phrases such as Atomgitter were acceptable to them. French language works, in contrast, had used réseau for the preceding century (except for Bravais himself), and this continues up to the modern day. By the 1910s, English speaking scientists had mostly abandoned Bravais' terminology of assemblage and followed the German usage, with space lattice closest in meaning to today's crystallographic lattice. In the next section we 
will move beyond the language to discuss how the idea of the physical and crystallographic lattice were understood by scientists.

\section{Changing ideas of the relationship between crystallographic lattice and crystal structure}

We have established in the previous section that the terms lattice energy, lattice dynamics etc at the point they were introduced, were in accord with the usage of lattice by crystallographers of the day. We will now discuss the evolution of the concept of the lattice and how it relates to physical components of the system. Recall that in the modern definition, a crystallographic lattice is an abstract object that represents the symmetry of a crystal. An important consequence of this is that a crystal structure can have only one lattice, just as it can have only one space group, or a molecule only one point group. With that idea in mind, we can try to ascertain how far back in history the idea existed.

Haïy proposed at the end of the $18^{\text {th }}$ century that the building blocks of crystals were solid polyhedral molecules. ${ }^{18}$ Delafosse, the student of Haüy, emphasised the difference between what were then termed the integrant molecule and the physical molecule. ${ }^{34}$ Bravais interpreted lattice nodes as coincident with centres of gravity of 'crystal elements' which might be taken to be molecules, or certainly a physical entity. ${ }^{14}$ Kelvin wrote in 1894 "Every crystal is a homogeneous assemblage of small bodies or molecules" using Bravais' term (assemblage) for a lattice. ${ }^{35}$ Fedorov wrote (translated from German) in 1892 that

"This is to be assumed as generally known [that] a point on the space lattice is replaced by a point group or molecule. A molecular lattice emerges from a point lattice, the symmetry of which is never higher than the symmetry of the associated space lattice ${ }^{132}$

NB Fedorov's use of Molekelgitter, molecular lattice, in contravention of the modern crystallographic convention.

Whether the objects were atoms or molecules or something else was not always agreed upon, but certainly towards the end of the $19^{\text {th }}$ century, the idea that lattices were linked with the arrangement of atoms or molecules in a crystal was widespread, if not universally accepted. However, we find the idea that the role of the lattice is to designate the overall symmetry of a 
crystal (as in the modern crystallographic usage) was not in place at this stage. Two quotes help illustrate this. Firstly, Groth in 1904 stated that a crystal: ${ }^{40}$

"consists of $\mathrm{n}$ interpenetrating regular point systems, each of which is formed from similar atoms; each of those point systems is built up from interpenetrating space lattices, each of the latter being formed from similar atoms occupying parallel positions."

Secondly, Tutton, writing in 1912, similarly describes the relationship between crystallographic lattice and atoms as follows: ${ }^{30}$

[The Bravais space lattice] is the structural foundation of the more complicated regular point-system according to which the crystal is homogeneously built up, and the points of which (the point-system) represent the chemical elementary atoms.

Whilst in Tutton's words there seems to be the kernel of the modern definition of crystallographic lattice, for Groth's there is no interpretation other that the space lattices are formed from the atoms. Such a formulation leaves little room for atoms away from lattice points or lattice points away from atoms, as we require in the modern definition. Groth goes further, suggesting that a compound crystal, made of several elements, is best considered as having one lattice for each kind of atom. As we have mentioned, this is fundamentally incorrect form a modern perspective: a crystal structure must have only one lattice by necessity.

Once X-ray diffraction was discovered in 1912 in Munich, very rapid progress was able to be made determining crystal structures, using the lattice theory and mathematical crystallography as the foundations. ${ }^{30}$ However, even in the first years following the discovery of diffraction, the distinction between the crystallographic lattice and the physical components of the crystal was not strongly made by the leading authors. A perfect example is W.H. and W.L. Bragg's determination of the structure of diamond, reported in 1913. ${ }^{4}$ They describe the arrangement of carbon atoms as follows:

the carbon atoms are not arranged on a space lattice, but they may be regarded as situated at the points of two interpenetrating face-centred space lattices. These lattices are so situated in relation to each other that, calling them $A$ and B, each point of lattice 
$B$ is surrounded symmetrically by four points of lattice A, arranged tetrahedron wise and vice versa

In fact, this idea of multiple lattices, one for each atom type, was used by W.H. and W.L. Bragg for several of the first structure solutions, e.g. $\mathrm{NaCl}$ and $\mathrm{ZnS}$. W.H. Bragg goes further, emulating Groth by writing equations that explicitly relate the number of atoms per unit volume to the number of lattices in the crystal. ${ }^{41}$

Ten years later in 1923, Ewald, wrote about how the advent of X-ray diffraction had in fact validated Groth's ideas on interpenetrating lattices. Noting Bragg's early efforts to interpret experimental data, he wrote: ${ }^{33}$

"the simplest type of lattice, the translation lattice, is not sufficiently general to explain the interferences [in diffraction], but rather the interlocking of such lattices, be it of the same type or of different atoms, must be considered. This is in full agreement with Groth's view of the crystal structure" (p. 84)

He went on to describe many crystal structures as consisting of interpenetrating, or nesting, lattices. For example, $\mathrm{Cu}_{2} \mathrm{O}$ is

“...described as a nesting of body centred $\mathrm{O}$ and face centred $\mathrm{Cu}$ lattices. Both lattices have the same cube edge." (p 158)

As we have seen, the idea of a crystal structure being made of interpenetrating lattices exists from the 1890s (according to Ewald it was proposed by Groth) and persists in the work of leading crystallographers until the 1920s, surviving even the revolution of the discovery of diffraction. However, it contrasts so sharply with the modern idea of the crystallographic lattice that Nespolo stated in 2019, concerning the description of the structure of diamond, that "interpenetrating two lattices does not make any sense". 5 That a widely accepted concept by crystallographers in 1923 now makes no sense to modern eyes is a clear indication that a major change has occurred in the meaning of lattice in the intervening period.

We believe the key to this transformation of the concept of the lattice lies in interpretation of diffraction patterns. W.H. Bragg observed in 1914 that "Generally, interpenetrating similarly 
placed lattices give a first-order spectrum [diffraction pattern] in the same place [the same diffraction angles] as any one of the lattices would do alone" 41 To illustrate this we show in Figure 2 the simulated powder $\mathrm{X}$-ray diffraction pattern for $\mathrm{NaCl}$. This structure might be described (as it was by Bragg, and had been guessed at by Groth and Barlow earlier) as consisting of interpenetrating face centred cubic lattices of sodium and chlorine. We also show in Figure 2 the simulated pattern that would arise if somehow one of these two atomic lattices were removed, leaving the other unchanged (for example if all the sodium were removed from the structure). As can be seen, the positions of the peaks, that is, the diffraction angles, do not change at all. Only the diffracted intensities change, as Bragg had observed.

The crucial point now emerges. While it can be seen that the description of the crystal structure can sensibly be made up of several atomic lattices combined, the diffraction pattern itself can be interpreted using just one. While Bragg in 1915 still used the terminology of separate lattices for different atoms, he emphasised now that X-ray diffraction gave two pieces of information separately: "the form of [the] fundamental lattice" which was obtained from the position of the diffraction spots, and the location of the atoms, which was obtained with more difficulty from the intensities. ${ }^{42}$ 


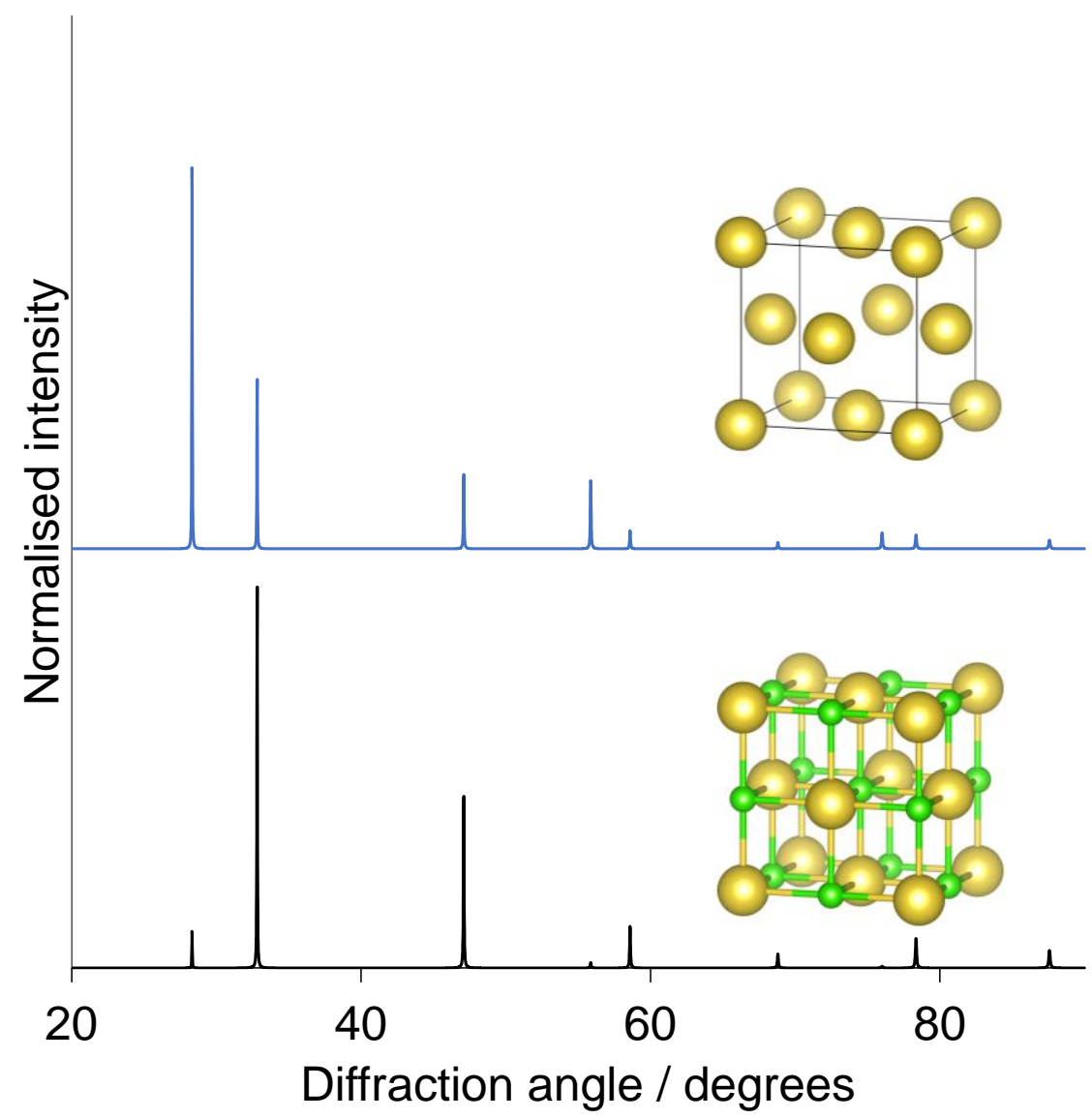

Figure 2. Simulated powder diffraction pattern (with $\mathrm{Cu}$ radiation) of $\mathrm{NaCl}$ (bottom) and a hypothetical structure where all of the sodium ions have been removed from $\mathrm{NaCl}$, leaving the chloride ions unaffected (top). This operation leaves the angles of the diffraction peaks unchanged, but the intensities change considerably, as pointed out by W.H. Bragg in $1914 .{ }^{41}$

The same result would be seen if instead the chlorine ions had been removed.

Bragg's observation (which was also made by others), and its interpretation not only unlocked crystal structure determination but points towards the practical use of conceptually separating the lattice from the atoms. The analysis of diffraction data could best be understood by considering the fundamental lattice (which affects the angles of diffraction) and the atoms (which affect the intensities of diffraction) as distinctly separate things. Whist it is perfectly clear to describe the calcium fluoride (fluorspar or fluorite) structure as consisting of two interpenetrating lattices, a face centred cubic lattice of calcium and a primitive cubic lattice of fluorine, if one is aiming to understand the diffraction pattern of fluorite that description is not very helpful. Better to assign what Bragg called a 'fundamental lattice', or what we call here a crystallographic lattice (or to modern crystallographers just 'lattice'). 
It is notable that for a few years, other crystallographers also adopted the use of the phrase fundamental lattice with this same meaning, ${ }^{43,44}$ and this can be seen as the recognition of the suddenly emergent need to move beyond the one-to-one correlation of atom with lattice point and structures made up of multiple lattices, to a description of structure more suited to interpretation of diffraction. We suggest, therefore, that it was the need to solve diffraction patterns of increasing complexity that made the sharp distinction between crystallographic and physical lattice of such importance. To put this another way, without the need to interpret diffraction patterns, there is no strong requirement to distinguish crystallographic from physical lattice, and this is why the crystallographers before 1912 did not do so.

\section{Discussion}

In the philosophy of classification, there are distinct theoretical views about which criteria should be used by scientists for the purposes of classification. Some theorists argue that classification is a matter of discovery of underlying composition. ${ }^{45}$ Classification can be considered an ontological project of defining the scientific terms in the right way so that they correspond to the natural classes that exist in the world, what can be called 'natural kinds' ${ }^{46}$ An alternative view argues that classes are stipulated according to the convention of use by scientists at a particular time. According to LaPorte, ${ }^{47}$ scientists have to make decisions about classifications, and sometimes prior usage of terms is vague, or may be used differently by different groups of scientists or indeed change radically over time. Often, in scientific practice this is done by reflective practice on the part of key stakeholders, until consensus is achieved.

The usage of the word lattice bridges applied mathematics and physical science in an interesting way. Gian-Carlo Rota argues that in mathematics, precise definitions emerge after concepts have been described, and even used, for perhaps many years. ${ }^{48}$ Carlo Cellucci expanded this concept and stated that, in mathematics: ${ }^{49}$

When what is defined is a concept which had previously been used without a precise definition, the definition contains an analysis of the concept, and may therefore express an advance in knowledge.

An example given by Cellucci is the definition of a sphere: Archimedes and Euclid defined the sphere differently, but equivalently: Archimedes as a locus of points equidistant from the 
centre, and Euclid as the figure inscribed by a rotating semi-circle. Even though these definitions are equivalent, Cellucci argues that in certain situations they can have different heuristic value, for example, Euclid's definition facilitates proving his proposition on the number of Platonic solids, which would be much more difficult using Archimedes' definition. ${ }^{50}$ We see a strong similarity with descriptions of crystal structure. As mentioned above, while the structure of fluorite, $\mathrm{CaF}_{2}$, can be described equivalently either as interpenetrating lattices made of $\mathrm{Ca}$ and $\mathrm{F}$ atoms, or as a single face centred cubic crystallographic lattice with an attached $\mathrm{CaF}_{2}$ motif, only the latter description can easily be used to understand diffraction patterns, and for that purpose, it has a greater heuristic value.

In contrast to the stipulative form of definition in mathematics, in physical science, an operational definition, one that defines a concept by how it is to be measured, is seen as the strongest form, as it relates the concept directly to physical phenomena, rather than defining it in terms of other concepts. ${ }^{51,52}$ The motivation of many of those who developed the mathematical concept of lattices in the $19^{\text {th }}$ century was a physical description of an as-yet unmeasurable physical reality: the arrangement of particles in a crystal. We have seen that the widespread assumption was that the purely mathematical lattice in fact corresponded to a real physical thing, and we have argued in the previous section that before X-ray diffraction, there was no real effort to distinguish the abstract lattice from the real particles. Despite this, the mathematical lattice, like many mathematical definitions, was a stipulated one. Once atomic positions could be experimentally measured by diffraction, the lattice could in fact obtain an operational definition: it was possible to write down a set of steps to interpret an experimental diffraction pattern in terms of the crystallographic lattice (Bragg's fundamental lattice). In doing so, the lattice necessarily became divorced from the physical particles, and became an abstract descriptor of overall symmetry of the crystal. Thus we can see a curious sequence of events: during the period when a lattice was defined only by mathematics (before 1912), it was seen as interchangeable with or identical to the physical particles. But once an operational definition was achieved and the lattice could be, in some sense, measured, it became entirely abstract. This change was driven by the advance in knowledge provided by X-ray diffraction.

We can now briefly revisit the possible justification for the claim, set out in the previous section, that the crystallographic definition of lattice is the correct one. 
Firstly, we can deal with the claim that the IUCr definition should take precedence. The chronological priority of this definition is challenged simply by noting that Born and coworkers defined lattice energy in 1918 and lattice dynamics in 1915 whereas the IUCr was founded in 1946. Of course, it can be argued that IUCr was simply formalising an already existing definition, so we can first consider whether the use of the terms lattice energy and lattice dynamics was appropriate by Born and coworkers in the 1910s. As we have discussed in section 3, it is clear that the usage of Gitter to mean a physical object was consistent with the leading crystallographers of the day. For the use of lattice energy to be an error of terminology by Born, it must be the case that leading crystallographers, including established mathematical and practical crystallographers such as Fedorov who discovered the space groups, Groth who wrote many textbooks and edited the leading journal for decades, the Braggs who first solved diffraction patterns, Ewald who co-founded the IUCr, and many others are wrong about such basic terminology of their specialist field. We find this idea untenable. There is no doubt that in the $1910 \mathrm{~s}$, lattice was conflated with physical particles, by crystallographers and physicists alike. It is clear from the writings of Bragg, Ewald, Groth, Tutton and contemporaries, that the idea of conceptually separating the lattice from the atoms was simply not important before 1912, and after only developed, over a number of years, as a practical solution to the need to interpret diffraction patterns. Thus, we suggest that instead of the 'true' historical meaning of lattice being changed by physicists, in fact it is the crystallographic term that has changed in meaning. This should be born in mind when judging the validity of any insistence that the crystallographic definition is the only correct one.

We can also dismiss Buerger's implication (made in the 1940s) that the 'incorrect' use of lattice (to mean a physical thing) stems from ignorance of the mathematical basis of crystallography. While the idea that physicists of the period lacked sufficient knowledge of geometry and symmetry was held somewhat widely, and was for example expressed by W.L. Bragg, ${ }^{53}$ we have shown here with numerous examples that what Buerger terms 'incorrect' was in fact standard usage by leading crystallographers just two decades before he wrote. Furthermore, Buerger's criticism of physicists in general can hardly be applied to Max Born in particular, whose very success in formulating lattice dynamics was predicated on his knowledge of mathematical crystallography. 


\section{Current situation, and possible resolutions}

To close, we discuss the possible resolutions. There are three plausible options to proceed in the face of the issues described in this article. Firstly, option (a) is that scientists decide the term lattice requires two distinct classifications to account for the two different types of lattice. We have attempted this approach in this article, by using the terms physical lattice and crystallographic lattice, to denote the two concepts. These or similar terms could be introduced. Secondly, (b) scientists could take a monist approach; that one of these definitions is better and should be adopted universally - and the other usage changed. The third option (c) is to accept that there is vague usage and allow communities of practitioners and researchers to decide which is the most appropriate classification in a discipline specific way or even on a case-by-case basis.

There may be a pedagogic virtue to taking option (a), adopting this approach would clearly delineate the distinction between the two types of lattice and would correct prior vague and ambiguous usage across different groups. It would also necessitate revision of crystallographic, chemistry and physics textbooks, and new students must be taught that the old terminology no longer applies.

Alternatively, in taking option (b), we could decide that either one of the two definitions is correct and that the other must be changed. Either we decide that terms such as lattice energy are misleading and should be replaced, for example with structural energy, while the term lattice continues to hold its crystallographic meaning. Alternatively, it could be decided that lattice energy and related terms are correct, and the crystallographic lattice concept should be renamed, perhaps as Bravais lattice, space lattice, fundamental lattice or even assemblage (all with longer historical precedent than the simple lattice). This option is the most fraught, as it involves changing a long-standing definition from either crystallography or physics, whilst maintaining that the opposing definition as 'more correct'. This option too requires alteration of textbooks as with option (a).

Lastly, in option (c) scientists might decide to agree to differ, claiming that vague usage is appropriate, or at least more appropriate than the other two options outlined above. This last option is essentially the status quo, although scientists could still commit to clarifying in their 
texts (as a few do currently) that other usage of lattice does exist, and other authors may validly choose a different definition.

With these three options before us, we turn finally to the practical steps that might resolve the issue. History has shown us that this classificatory issue is not just a matter of scientific disagreement. At the root of this are two very different notions about how classification should be done in science; namely classification as the discovery of constitution as opposed to classification as a matter of stipulation by scientists. In the case at hand, it seems the prospect for solution through some new discovery looks very unlikely. If on the other hand, we think that the definition of lattice is to be stipulated, then a process should take place to decide on the best way to do this.

This kind of process is not unprecedented. Scientific consensus is widely used as a social indicator of the existence of knowledge in public forums. Consensus is also used by scientists as a methodology for decision making when there is a controversy or disagreement between practitioners. Indeed, this is enshrined in governance itself, for example, public bodies like the National Institute of Health (NIH) and the Intergovernmental Panel on Climate Change (IPCC) release consensus statements to provide authoritative answers to disputed questions.

However, we might think about how consensus is achieved in the physical sciences. Beaty has written extensively about the role of consensus among experts. ${ }^{54}$ Scientists often agree to jointly accept a position, despite disagreements among them. In such cases, not all experts agree with the position, but accept it as the rational position of the group.

One important example of consensus particularly relating to classification is the example of the declassification of Pluto as a planet. It is interesting to note the rationale for this reclassification by the planet definition committee claim:

"Such decisions and recommendations establish conventions that are meant to help our understanding of astronomical objects and processes."

Hence, recommendations for classificatory decision in this case rest on well-established scientific facts and have a broad consensus in the community concerned. 
Should a definitive resolution be desired, i.e. option (a) or (b) above, a similar process might be undertaken by the group of stakeholders who use lattice classificatory concepts. We think it is clear that unilateral definition by bodies representing either crystallographers or physicists (or chemists) will not solve this issue. Indeed, this has already been attempted directly by the IUCr, and also indirectly by IUPAC. The former defined lattice at some point after the 1940s, contrary to the several decades of differing prior usage of physicists, and indeed crystallographers. That many physicists and chemists today do not follow the IUCr definition may be a cause of frustration to some crystallographers, but to us does not seem surprising given the historical development of the term we have descried in this article. IUPAC maintains definitions including the term lattice that must refer to a physical object, which are objected to by some crystallographers. Neither of these approaches has solved the issue, and indeed they may make the situation worse by tending to polarise scientists in each field to support the definition of their own international body. A consensus conference would be one way to approach the issue, so that stakeholders could provide a rationale for taking one of the options (a-c) and discuss with a view to reaching consensus on how this classificatory issue might be resolved. 


\section{Conclusion}

In conclusion, we have addressed the classification of lattice within the community of scientists working on crystalline materials in crystallography, chemistry and physics. Whilst it has been stated for many years that the crystallographic definition of lattice is in some way more valid than the physical definition, our historical analysis shows that this issue is far from clear cut.

We find that the role of lattice as a descriptor of the symmetry of a crystal structure was only fully developed after the discovery of X-ray diffraction in 1912. At this time, the field of lattice dynamics was being established, and a host of new terminology, such as lattice energy and lattice vibration, was introduced which is still used today. In implementing this terminology, the pioneers of lattice dynamics followed the conventions of the crystallographers of the day, who readily used terms such as 'atomic lattice' and 'molecular lattice'. However, the terminology of the crystallographers changed over the following decades. We suggest here that this change was in response to the need to interpret diffraction patterns, which required the differentiation between what Bragg called the fundamental lattice and the atom positions. In this way the language of crystallographers and physicists diverged until the two meanings each built up a seemingly unassailable volume of usage which is a major challenge to any reconciliation. Such resolution may take the form of the current status quo, where two uses are acknowledged, but with perhaps a focus from scientists in all fields on mutual acceptance of the validity of each. Alternatively, a more definitive resolution may come about through consensus of stakeholders from a range of scientific disciplines.

\section{Acknowledgements}

The authors are grateful to Dr. Anna Regoutz and Dr Jeremy Karl Cockcroft (UCL), Prof. Eric Scerri (UCLA) and Prof. Robin Hendry (University of Durham) for reading the manuscript and providing helpful comments. We also thank the UCL Science and Technology Studies seminar audience for feedback and suggestions.

\section{References}

(1) Wong, C. L.; Chu, H.-E.; Yap, K. C. Developing a Framework for Analyzing Definitions: A study of The Feynman Lectures. International Journal of Science Education 2014, 36 (15), 2481 . 
(2) Muller, P. Glossary of terms used in physical organic chemistry (IUPAC Recommendations 1994) J Pure and Applied Chemistry. 1994, 66 (5), 1077.

(3) Nespolo, M. Lattice versus structure, dimensionality versus periodicity: a crystallographic Babel? Journal of Applied Crystallography 2019, 52, 451.

(4) Bragg, W. H.; Bragg, W. L. The structure of the diamond. Proceedings of the Royal Society of London. Series A, Containing Papers of a Mathematical and Physical Character 1913, 89 (610), 277.

(5) Nespolo, M. The lattice sickness pandemic. IUCr Newsletter 2019, 27 (2).

(6) Born, M.; Huang, K. Dynamical theory of crystal lattices, Oxford University Press, 1954.

(7) Atkins, P. W. Shriver \& Atkins' inorganic chemistry; Oxford University Press: Oxford; New York, 2010.

(8) Klemens, P. G. Theory of lattice thermal conductivity: Role of low-frequency phonons. International Journal of Thermophysics 1981, 2 (1), 55.

(9) Simon, S. H. The Oxford solid state basics, Oxford University Press, 2016.

(10) Hoddeson, L.; Braun, E.; Teichmann, J.; Weart, S. Out of the Crystal Maze: Chapters from the History of Solid State Physics; Oxford University Press, 1992.

(11) Authier, A. Early Days of X-ray Crystallography; Oxford University Press, 2013.

(12) Glazer, A. M. Crystallography: A Very Short Introduction; Oxford University Press, 2016.

(13) Ewald, P. P. The myth of myths; comments on P. Forman's paper on "the discovery of the diffraction of X-rays in crystals". Archive for History of Exact Sciences 1969, 6 (1), 72.

(14) Kubbinga, H. Crystallography from Haüy to Laue: controversies on the molecular and atomistic nature of solids, Acta Crystallographica Section A 2012, 68 (1), 3.

(15) Hildebrandt, G. The Discovery of the Diffraction of X-rays in Crystals - A Historical Review, Crystal Research and Technology, 1993, 28 (6), 747.

(16) Wilkins, S. W. Celebrating 100 years of X-ray crystallography. Acta Crystallographica aFoundation and Advances 2013, 69, 1.

(17) Schwarzenbach, D. The success story of crystallography. Acta Crystallographica aFoundation and Advances 2012, 68, 57.

(18) Lalena, J. N. From quartz to quasicrystals: probing nature's geometric patterns in crystalline substances. Crystallography Reviews 2006, 12 (2), 125.

(19) Hilton, H. Mathematical Crystallography and the Theory of Groups of Movements, 1903.

(20) Gasman, L. D. Myths and X-rays. The British Journal for the Philosophy of Science 1975, 26 (1), 51 .

(21) Buerger, M. J. X-ray Crystallography: An Introduction to the Investigation of Crystals by Their Diffraction of Monochromatic X-radiation; J. Wiley \& Sons, Incorporated, 1942.

(22) Madelung, E. Molekulare Eigenschwingungen. Nachr. Ges. Wiss. Göttingen 1909, 304, 100.

(23) Born, M.; Lande, A. Uber die absolute Berechnung der Kristalleigenschaften mit Hilfe Bohrscher Atommodelle. Ber. Preuss. Akad. Wiss. 1918, 45, 1048.

(24) International Conference on Lattice, D.; Wallis, R. F.; institutet, H. C. Ø., Oxford; New York.

(25) Debye, P. Zur Theorie der spezifischen Wärmen. Annalen der Physik 1912, 344 (14), 789.

(26) Einstein, A. Die Plancksche Theorie der Strahlung und die Theorie der spezifischen Wärme. J Annalen Der Physik 1906, 327, 180.

(27) Blackman, M. On the Relation of Debye Theory and the Lattice Theory of Specific Heats. Proceedings of the Royal Society of London. Series A, Mathematical and Physical Sciences 1942, 181 (984), 58.

(28) Kemmer, N.; Schlapp, R. Max Born, 1882-1970. Biographical Memoirs of Fellows of the Royal Society 1971, 17, 17.

(29) Paufler, P. William Barlow's early publications in the 'Zeitschrift für Krystallographie und Mineralogie' and their influence on crystal structure research $J$ Zeitschrift für Kristallographie - Crystalline Materials. 2019, 234 (11-12), 769.

(30) Tutton, A. E. H. The Crystal Space-Lattice Revealed by Röntgen Rays. Nature 1912, 90 (2246), 306.

(31) Sohncke, L. Die regelmässigen ebenen Punktsysteme von ungebrenzter Ausdehnung. Journal für die reine und angewandte Mathematik 1874, (77), 47. 
(32) Fedorow, E. v. II. Zusammenstellung der krystallographischen Resultate des Herrn

Schoenflies und der meinigen J Zeitschrift für Kristallographie - Crystalline Materials. 1892, $20(1-6), 25$.

(33) Ewald, P. P. Kristalle und Röntgenstrahlen; Springer-Verlag Berlin Heidelberg, 1923.

(34) Delafosse, G. Recherches sur La Cristallisation, Impr. Royale, Paris, 1843.

(35) Kelvin, L. The Molecular Tactics of A Crystal, Oxford University Press, 1894.

(36) Barlow, W. A Mechanical Cause of Homogeneity of Structure and Symmetry Geometrically Investigated; with special application to Crystals and to Chemical Combination. Proceedings of the Physical Society of London 1897, 16 (1), 54.

(37) Barlow, W. On the interpretation of the indications of atomic structure presented by crystals when interposed in the path of X-rays. 1914, 91 (623), 1.

(38) Bragg, W. L.; Bragg, W. H. The structure of some crystals as indicated by their diffraction of X-rays. 1913, 89 (610), 248.

(39) Bragg, W. L.; Bragg, W. L.; Phillips, D. C.; Lipson, H. The Development of X-ray Analysis; G. Bell, 1975.

(40) Groth, P. On Crystal Structure and its Relation to Chemical Constitution. Report Of The Seventy Fourth Meeting Of The British Association 1904.

(41) Bragg, W. H. The X-ray Spectra Given by Crystals of Sulphur and Quartz. Proceedings of the Royal Society of London. Series A, Containing Papers of a Mathematical and Physical Character 1914, 89 (614), 575.

(42) Bragg, W. H. IX. Bakerian Lecture; X-rays and crystal structure. Philosophical Transactions of the Royal Society of London. Series A, Containing Papers of a Mathematical or Physical Character 1915, 215 (523-537), 253.

(43) Bernal, J. D.; Bragg, W. L. The structure of graphite. Proceedings of the Royal Society A, 1924, 106 (740), 749.

(44) Wyckoff, R. W. G. The Crystal Structure of Cesium Dichloroiodide,. J. Am. Chem. Soc. 1920, $42(6), 1100$.

(45) Hendry, R.; xa; Findlay. Elements, Compounds, and Other Chemical Kinds. Philosophy of Science 2006, 73 (5), 864.

(46) Tobin, E.; Bird, A. In Stanford Encyclopedia of Philosophy; Zalta, E. N., Ed., 2009.

(47) LaPorte, J. Natural Kinds and Conceptual Change; Cambridge University Press: Cambridge, 2003.

(48) Rota, G.-C. Indiscrete Thoughts; Birkhauser, 1997.

(49) Cellucci, C. Indiscrete Variations on Gian-Carlo Rota's Themes Invited Chapter, In: Damiani E., D’Antona O., Marra V., Palombi F. (eds) From Combinatorics to Philosophy. Springer, Boston, 2009

(50) Cellucci, C. Definition in mathematics. European Journal for Philosophy of Science 2018, 8 (3), 605.

(51) Slisko, J.; Dykstra, J., Dewey I. The role of scientific terminology in research and teaching: Is something important missing? Journal of Research in Science Teaching, 1997, 34 (6), 655.

(52) Angeles, P. A. A Dictionary of Philosophy, HarperCollins, 1981.

(53) Bragg, W. L. The Rutherford Memorial Lecture, 1960 - The development of X-ray analysis. Proceedings of the Royal Society A, 1961, 262 (1309), 145.

(54) Beatty, J.; Moore, A. Should We Aim for Consensus? Episteme 2010, 7 (3), 198. 